\title{
Miniaturized Electrochemical Sensors to Monitor Fetal Hypoxia and Acidosis in a Pregnant Sheep Model
}

\author{
Míriam Illa ${ }^{1,2, *, \dagger}$, Laura Pla ${ }^{1,+} \oplus$, Sergio Berdún ${ }^{1}\left(\mathbb{D}\right.$, Mònica Mir $3,4,5 \oplus$, Lourdes Rivas ${ }^{4}$, Samuel Dulay ${ }^{4}$, \\ Nicole Picard-Hagen ${ }^{6}{ }^{(}$, Josep Samitier ${ }^{3,4,5}{ }^{(}$, Eduard Gratacós ${ }^{1,2,7,8}$ and Elisenda Eixarch $1,7,8(\mathbb{D}$ \\ 1 BCNatal I Fetal Medicine Research Center (Hospital Clínic and Hospital Sant Joan de Déu), \\ Universitat de Barcelona, 08028 Barcelona, Spain; laura.pla.codina@gmail.com (L.P.); \\ sberdun@pcb.ub.es (S.B.); egratacos@sjdhospitalbarcelona.org (E.G.); EIXARCH@clinic.cat (E.E.) \\ 2 Institut de Recerca Sant Joan de Déu, 08950 Esplugues de Llobregat, Spain \\ 3 Centro de Investigación Biomédica en Red en Bioingeniería, Biomateriales y Nanomedicina (CIBER-BBN), \\ Monforte de Lemos 3-5, Pabellón 11, 28029 Madrid, Spain; mmir@ibecbarcelona.eu (M.M.); \\ jsamitier@ibecbarcelona.eu (J.S.) \\ 4 Nanobioengineering Group, Institute for Bioengineering of Catalonia (IBEC), Baldiri Reixac 15-21, \\ 08028 Barcelona, Spain; lrivas@ibecbarcelona.eu (L.R.); dulaysamuel@yahoo.com (S.D.) \\ 5 Department of Electronics and Biomedical Engineering, University of Barcelona, Martí i Franquès 1, \\ 08028 Barcelona, Spain \\ 6 ToxAlim (Research Centre in Food Toxicology), Université de Toulouse, INRAE, ENVT, INP-Purpan, UPS, \\ 31027 Toulouse, France; nicole.hagen@envt.fr \\ 7 Institut d'Investigacions Biomèdiques August Pi i Sunyer (IDIBAPS), 08028 Barcelona, Spain \\ 8 Centre for Biomedical Research on Rare Diseases (CIBER-ER), 08028 Barcelona, Spain \\ * Correspondence: MIRIAMIL@clinic.cat; Tel.: +34-934-487-496 \\ check for \\ updates \\ + These authors contributed equally to this work.
}

Citation: Illa, M.; Pla, L.; Berdún, S.; Mir, M.; Rivas, L.; Dulay, S.; Picard-Hagen, N.; Samitier, J.; Gratacós, E.; Eixarch, E. Miniaturized Electrochemical Sensors to Monitor Fetal Hypoxia and Acidosis in a Pregnant Sheep Model. Biomedicines 2021, 9, 1344. https://doi.org/ 10.3390/biomedicines 9101344

Academic Editor: Sharon Marx

Received: 2 September 2021

Accepted: 23 September 2021

Published: 28 September 2021

Publisher's Note: MDPI stays neutral with regard to jurisdictional claims in published maps and institutional affiliations.

Copyright: (c) 2021 by the authors. Licensee MDPI, Basel, Switzerland. This article is an open access article distributed under the terms and conditions of the Creative Commons Attribution (CC BY) license (https:// creativecommons.org/licenses/by/ $4.0 /)$.

\begin{abstract}
Perinatal asphyxia is a major cause of severe brain damage and death. For its prenatal identification, Doppler ultrasound has been used as a surrogate marker of fetal hypoxia. However, Doppler evaluation cannot be performed continuously. We have evaluated the performance of a miniaturized multiparametric sensor aiming to evaluate tissular oxygen and $\mathrm{pH}$ changes continuously in an umbilical cord occlusion (UCO) sheep model. The electrochemical sensors were inserted in fetal hindlimb skeletal muscle and electrochemical signals were recorded. Fetal hemodynamic changes and metabolic status were also monitored during the experiment. Additionally, histological assessment of the tissue surrounding the sensors was performed. Both electrochemical sensors detected the $\mathrm{pO}_{2}$ and $\mathrm{pH}$ changes induced by the $\mathrm{UCO}$ and these changes were correlated with hemodynamic parameters as well as with $\mathrm{pH}$ and oxygen content in the blood. Finally, histological assessment revealed no signs of alteration on the same day of insertion. This study provides the first evidence showing the application of miniaturized multiparametric electrochemical sensors detecting changes in oxygen and $\mathrm{pH}$ in skeletal muscular tissue in a fetal sheep model.
\end{abstract}

Keywords: umbilical cord occlusion; continuous monitoring of acid-base status; high-risk pregnancies; electrochemical sensors

\section{Introduction}

Perinatal asphyxia is a major cause of severe brain damage and death during the perinatal period. While acute asphyxia affects 1 in every 1000 live births in developed countries [1], chronic forms of fetal hypoxia may occur in up to 1 to $3 \%$ of pregnancies. The main causes of chronic fetal hypoxia are complications related to placental insufficiency, namely preeclampsia and fetal growth restriction. Irrespective of the cause, chronic hypoxia generally results from insufficient oxygen supply to the fetus, eventually leading to hypoxia, hypercapnia, and acidosis. In a primary phase, fetal circulatory and non-circulatory 
adaptive mechanisms try to cope with asphyxia and preserve vital organ function. However, in severe and/or prolonged insults, compensatory mechanisms fail, leading to cell death via necrosis and apoptosis [2].

Close monitoring of pregnancies with placental insufficiency is based on Doppler evaluation of fetal hemodynamics [3]. Changes in the hemodynamics of fetal blood flow in the ductus venosus or in the umbilical artery are highly suggestive of progressive hypoxia and eventually of fetal acidemia [4], and Doppler is used to decide elective delivery when the risk of fetal death or brain damage is deemed too high [5]. Since fetuses with severe forms of placental insufficiency are normally extremely premature, timing of delivery is critical to avoid fetal death but prolong pregnancy as much as possible to minimize the neonatal complications of prematurity.

While Doppler ultrasonography has been an invaluable tool to improve the timing of delivery in critically ill, very preterm fetuses, it is still limited by the impossibility of performing continuous monitoring. The development of a system to monitor acid-base status continuously would be a great step forward in the clinical management of very high-risk pregnancies. Previous research has demonstrated the usefulness of oxygen and $\mathrm{pH}$ sensors to monitor acid-base status continuously in different tissues. Oxygen sensors based on either pulse-oximetry or fiber-optic sensing inserted in fetal tissue during labor in humans or placed endovascularly during endoscopic fetal surgery in sheep have shown promising results in detecting oxygen variations [5-8]. Similarly, sensors able to monitor the levels of fetal pH have also been described [8-11]. Despite all these advances, these sensors are not suitable for insertion in early pregnancy, due to the need to protect the uterus and the very small size of fetal tissues. In this regard, miniaturized electrochemical sensors insertable through a needle would be required in order to monitor fetal acid-base status. Previous studies from our group have demonstrated that miniaturized electrochemical sensors could monitor oxygen or $\mathrm{pH}$ changes continuously in a model of ventilatory hypoxia in adult rabbits [12-14]. The development of a multiparametric electrochemical sensor aiming to monitor oxygen and $\mathrm{pH}$ at the same time would offer a great advantage in the monitoring of these pregnancies.

In this study, we aimed to test the performance of integrated and miniaturized $\mathrm{pH}$ and oxygen sensors to enable implantation for fetal monitoring and to detect changes in these parameters simultaneously in a lamb model of acute hypoxia $[15,16]$. We have used a sequential UCO to induce fetal acid-based metabolite alterations in a gradual and controlled manner and the electrochemical sensor signals, blood test analyses, and Doppler hemodynamics were analyzed.

\section{Materials and Methods}

\subsection{Animals: Ethics, Housing, and Preparation}

A total of eight Ripollesa pregnant ewes with gestational ages between 115-125 days (term $=147-150$ days) and weight ranging from 60 to $80 \mathrm{~kg}$ were included in this study. Five of the eight ewes carried singletons and three of the eight carried twins. The animals were provided by a certified commercial farm (Animal Bianya, Catalonia, Spain) and were acclimated for two weeks before surgery. Their clinical condition was assessed by veterinary staff before starting the study. The animals were housed following local standards. Ewes were food fasted $24 \mathrm{~h}$ before the surgery and water fasted $12 \mathrm{~h}$ before the surgery. Animal handling and all experimental procedures were performed in accordance with applicable regulations and guidelines and with the approval of the Animal Experimental Ethics Committee of the Universitat de Barcelona (Ref. 214.17) and the competent authority Generalitat de Catalunya (Ref. 9645).

\subsection{Miniaturized Electrochemical Sensors: $\mathrm{pH}$ and $\mathrm{pO}_{2}$}

The sensors used in this article were developed and optimized in previous work $[13,14]$. In previously reported articles, the sensitivity, specificity, reproducibility, and response time of these sensors are described, which were validated in adult rabbits. In the present 
work, difficulties in fetal monitoring are faced, such as the smaller size for the insertion of sensors, more instability in vivo, lower oxygen content in fetal blood, and lower changes of analytes in tissue after ischemia. For fetal application, the sensors were fabricated to be longer $(1 \mathrm{~m})$. For that reason, the insulating layer of one end of the metallic wires ( $\mathrm{Pt}$ or $\mathrm{Ag}$, $15 \mathrm{~cm}$ in length and $125 \mu \mathrm{m}$ in diameter) was carefully removed to solder it with a copper wire (1 $\mathrm{m}$ in length). Once the microsoldered section had been isolated, the modifications for preparing the working and the reference electrodes for both types of electrochemical sensor were performed as previously described $[13,14]$. After the sensors were individually functionalized, they were integrated into a biocompatible tube with a final diameter of $500 \mu \mathrm{m}$ (See supporting information Figure S1).

\subsection{Animal Instrumentation}

\subsubsection{Ewe Surgery}

The animals were premedicated with ketamine, xylazine, and midazolam $(4 \mathrm{mg} / \mathrm{kg}$, $0.2 \mathrm{mg} / \mathrm{kg}$, and $0.2 \mathrm{mg} / \mathrm{kg}$ respectively, IM). For induction anesthesia, $2-4 \mathrm{mg} / \mathrm{kg}$ (IV) of propofol was administered. An endotracheal tube was then inserted, and mechanical pressure-controlled ventilation was started. Anesthesia was maintained with isoflurane $(1.5-3 \%)$ using a semi-closed system. Heart rate, respiratory rate, oxygen saturation, endtidal carbon dioxide, and reflexes were monitored every 15-30 min. Body core temperature was maintained using an electric pad. Before the start of surgery, $1 \mathrm{~L}$ of saline $0.9 \% \mathrm{NaCl}$ was infused using a jugular catheter, and buprenorphine $(0.02 \mathrm{mg} / \mathrm{kg}, \mathrm{IV})$, and progesterone (150 mg, SC) were also administered. Urinary tract cannulation and orogastric intubation were performed.

Before the start of the surgery, the animals were prepared for aseptic surgery and $10 \mathrm{~mL}$ of lidocaine ( $2 \%$ ) were injected intradermally on the site of the abdominal incision. A $15-\mathrm{cm}$ infraumbilical midline laparotomy was performed for uterus exteriorization. The number of fetuses, their position, the fetal heart rate (FHR), and deepest vertical pocket (DVP) of amniotic fluid were recorded using ultrasonography (VIVIQ Q, lineal probe, 7.5 MHz) before hysterotomy. In twin gestations, only one uterine horn was exposed and used for the experiment. The fetal position was identified and a 6-8 $\mathrm{cm}$ hysterotomy was performed using a running suture on both incisional sides for fetal membrane fixation (poliglecaprone, $2 / 0$ ). During the surgery, fluidotherapy with saline $0.9 \% \mathrm{NaCl}$ solution was administered $(500 \mathrm{~mL} / \mathrm{h}$ ) and serial maternal gasometry from the jugular vein (between 2 and 4 samples per experiment) was performed throughout the experiment in order to evaluate maternal acid-base status (EPOC reader and EPOC BEGM test card, Alere/Siemens healthcare, Barcelona, Spain).

\subsubsection{Fetal Instrumentation}

Fetal hindlimbs were exteriorized through hysterotomy, and fentanyl ( $0.2 \mathrm{~mL}, \mathrm{IM})$ was administered for fetal anesthesia. An incision of $3-4 \mathrm{~cm}$ in the right inguinal zone was performed for the fetal iliac artery dissection. Catheterization of the iliac artery was performed for all the included fetuses $(n=8)$ with a thin catheter $(0.75 \mathrm{~mm}$ of internal diameter and $1.45 \mathrm{~mm}$ of external diameter) and was used for serial fetal blood sampling in order to evaluate acid-based metabolites (using the EPOC reader and EPOC BEGM test card, Alere/Siemens Healthcare, Barcelona, Spain). The catheter was fixed with simple knots (silk, 3/0) and fetal skin was closed with a running suture (polyglycolic acid, 3/0). Thereafter, 1-2 oxygen and 1-2 pH electrochemical sensors were inserted in the right and left femoral fetal quadriceps, respectively. We did a 1-cm skin incision and the femoral quadriceps was exposed. Electrochemical sensors were implanted through a 2-3 mm muscle incision and secured to the parenchyma using simple knots (silk, 3/0). One to two oxygen or $\mathrm{pH}$ electrochemical sensors were inserted in each animal. The skin was closed using a running suture (polyglycolic acid, 3/0). Afterwards, the umbilical cord was also exteriorized through hysterotomy and a vascular occluder (OC20HD, UNO Roestvaststaal BV, Zevenaar, Netherlands) connected to a $10 \mathrm{~mL}$ syringe loaded with 
saline was placed around the umbilical cord. Finally, fetal hind limbs and umbilical cord were interiorized inside the uterus. An amniotic catheter was also inserted through the hysterotomy in order to infuse warm $\mathrm{NaCl} 0.9 \%$ to maintain the temperature and amniotic volume throughout the experiment. The amniotic cavity insufflation was performed until the initial DVP was reached (approximately $150 \mathrm{~mL}$ ). Sensor wires, iliac vascular catheter, amniotic catheter, and the occluder tubing were exteriorized through the uterine incision. Hysterorraphy was then performed using a running suture (polidioxanone, $3 / 0$ ). The electrodes of the electrochemical sensors were connected to a portable electrochemical device for the continuous monitoring of the signals of the oxygen and $\mathrm{pH}$ electrochemical sensors. During the experiment, the uterus was kept outside the abdominal cavity and was continuously rinsed with warm $\mathrm{NaCl} 0.9 \%$ and temperature controlled with an external thermal lamp.

\subsection{Occlusion Protocol and Monitoring}

The occlusion protocol was divided into four different stages: (1) basal; (2) 50\% occlusion; (3) 100\% occlusion; and (4) recovery. For the basal and recovery stages, the occluder was deflated and for the occlusion periods, the occluder was inflated with $1.5 \mathrm{~mL}$ or $3 \mathrm{~mL}$ of saline in order to obliterate $50 \%$ or $100 \%$ of the umbilical cord, respectively, and reduce the blood flow. In each stage, fetal blood sampling and electrochemical sensor signals (oxygen and $\mathrm{pH}$ ) were recorded at the same moment in 5-to-10-min intervals. For blood sampling analyses, $0.2 \mathrm{~mL}$ of blood from the fetal iliac artery catheter was obtained for fetal gasometry with EPOC system including $\mathrm{pO}_{2}, \mathrm{pH}$, bicarbonate, potassium, and lactate. Additionally, fetal Doppler evaluation was also recorded in each stage using VIVID Q ultrasound equipment (GE Healthcare, Chalfont Saint Giles, UK) including (i) FHR; (ii) pulsatility index (IP) of the umbilical artery (UAPI) in the perivesical portion; (iii) IP of the ductus venosus (DVPI); (iv) maximum blood flow velocity $(\mathrm{cm} / \mathrm{s})$ in the umbilical vein (UV) and diameter $(\mathrm{mm})$ of the UV in the intrabdominal portion of the vessel. For each stage, at least one measurement was obtained in each period.

\subsubsection{Basal Stage}

After finalizing fetal instrumentation and hysterorraphy, basal registration was then started for $10 \mathrm{~min}$. During that time, two measurements of fetal and maternal gasometry and fetal sensor signaling (in $\mathrm{nA}$ from the oxygen sensor and $\mathrm{mV}$ from the $\mathrm{pH}$ sensor) were recorded.

\subsubsection{Occlusion Stage}

The $50 \%$ occlusion stage lasted $20 \mathrm{~min}$, while the $100 \%$ occlusion stage lasted $10 \mathrm{~min}$. During these periods, regular fetal gasometry was performed every $10 \mathrm{~min}$ in the $50 \%$ occlusion stage and every $5 \mathrm{~min}$ in the $100 \%$ occlusion stage. At the same time, sensor signaling (in $\mathrm{nA}$ from the oxygen sensor and $\mathrm{mV}$ from the $\mathrm{pH}$ sensor) was also recorded.

\subsubsection{Recovery Stage}

After 10 min of $100 \%$ occlusion, the occluder was deflated and the recovery stage was started, lasting $20 \mathrm{~min}$. Fetal gasometry and electrochemical sensor recording was obtained every 10 min during this stage.

\subsection{Sampling and Histological Analyses}

After the occlusion protocol, the animals were sacrificed with pentobarbital $(200 \mathrm{mg} / \mathrm{kg}$, IV) administrated to the ewe through the jugular vein. Death was confirmed by the cessation of circulation and breathing in both ewes and fetuses. Muscular tissue surrounding the insertion area of the electrochemical sensors inserted was carefully excised; fixed for one week by immersion in $10 \%$ buffered formalin and embedded in paraffin for further histological analyses. Paraffin blocks were serially cut in $5 \mu \mathrm{m}$ thick transverse sections with a microtome and standard hematoxylin/eosin (Mayer's Hematoyxlin, Ref. 51275 
Sigma-Aldrich, Saint Louis, MO, USA; Eosin, Ref. 1.15935.0100 Merk, Darmstadt, Germany) and masson trichrome staining (Ref. HT15-1KT, Sigma-Aldrich, Saint Louis, MO, USA) were performed. One representative section from each sample was examined under an optic microscope (CH-9435, type DFC425C, Leica Microsystems, Hospitalet de Llobregat, Spain). Tissue integrity and cell infiltration were analyzed with hematoxylin eosin staining, whereas masson trichome staining was used for the evaluation of fibrotic reaction. The design of this study is summarized in Figure 1.
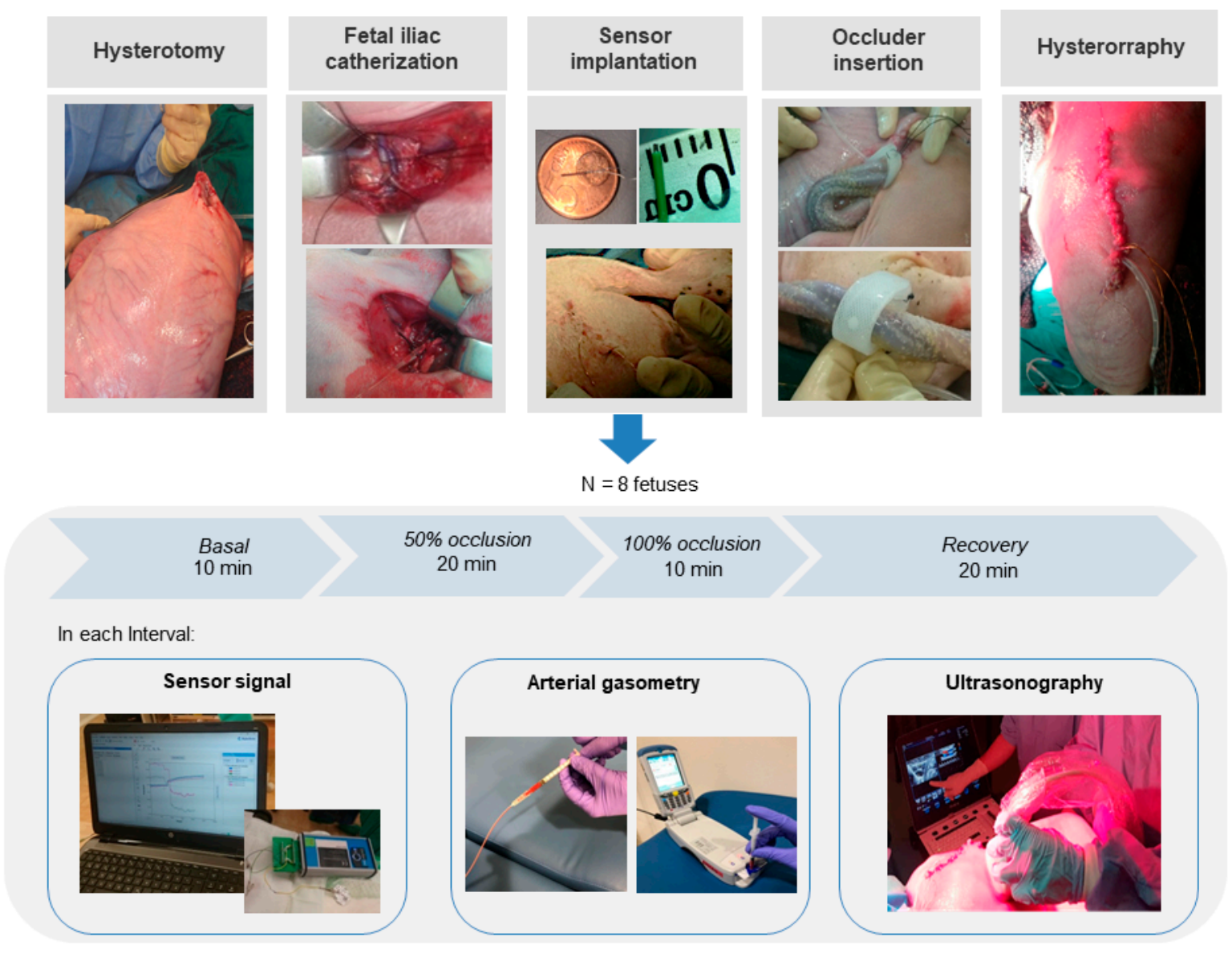

Figure 1. Illustrative images of the study design.

\subsection{Statistical Analyses}

The data were expressed as mean and standard error of the mean (SEM). A one-way ANOVA analysis with a Dunnett's post-test was used for the analysis of the evolution of acid-based metabolite results, sensor parameters, and Doppler results during the UCO. Additionally, the association between electrochemical sensing results with the $\mathrm{pO}_{2}$ and $\mathrm{pH}$ values and Doppler results was analyzed by means of Pearson's correlation analysis. Statistical significance was declared at $p<0.05$ in all variables evaluated. All statistical analysis was performed using GraphPad Prism 6.0 software (San Diego, CA, USA).

\section{Results}

\subsection{Acid-Based Metabolite Results}

Of the eight fetuses included in the study, one was excluded due to anesthesia problems that led to maternal acidosis during the basal period (maternal $\mathrm{pH}$ of 7.16). No other surgical or anesthesia complications were observed, leaving a final sample of seven fetuses included in the study. Maternal gasometry of the included animals was within normal ranges during all the different stages of the experiment. 
A description of the metabolite results obtained by fetal blood sampling during the different phases of the study is detailed in Figure 2. During the hypoxia-acidosis periods, a significant decrease in $\mathrm{pO}_{2}, \mathrm{pH}$, and bicarbonate and a significant increase in lactate and potassium concentration in comparison with the basal period were observed. The $\mathrm{pO}_{2}$ decrease was the quickest and presented a more pronounced change in comparison with the rest of the metabolites, which presented more progressive and less marked changes. The changes were more marked and quicker in the 100\% occlusion period. Finally, in the recovery stage, three of the seven fetuses did not overcome the fetal hypoxia-acidosis changes induced by the UCO and died during the recovery period. In these fetuses, the $\mathrm{pH}$ ranged between 6.69 and 6.77. The rest of the fetuses presented trends to an increase in $\mathrm{pH}$ levels without reaching the basal values (7.07). In the surviving animals, $\mathrm{pO}_{2}$ and $\mathrm{K}+$ were the only parameters in the recovery phase that reached similar levels to those reported in the basal period.
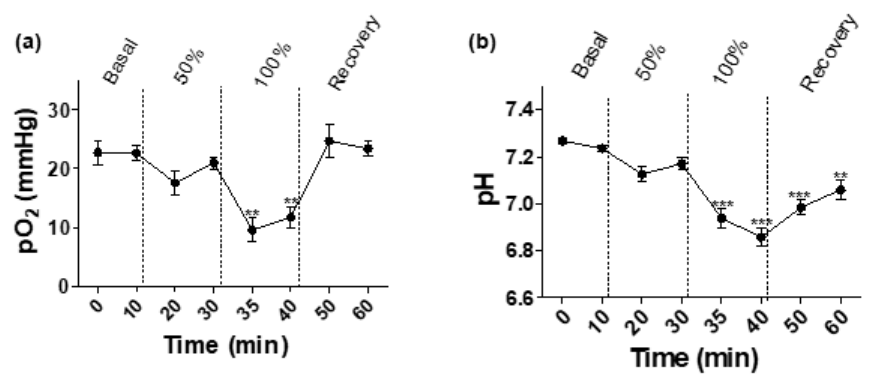

(c)

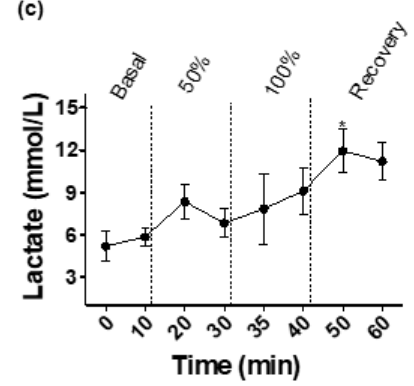

(d)

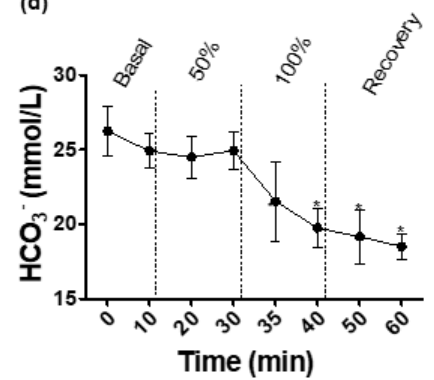

(e)

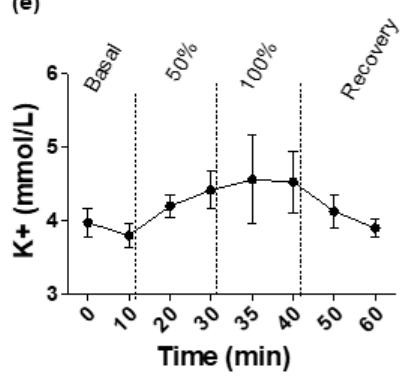

Figure 2. Evolution of arterial acid-based metabolites during the occlusion protocol (basal, 50\% occlusion, 100\% occlusion and recovery periods): (a) Partial pressure of oxygen $\left(\mathrm{pO}_{2}\right)$; (b) $\mathrm{pH}$; (c) lactate; (d) bicarbonate $\left(\mathrm{HCO}_{3}{ }^{-}\right)$; and (e) potassium $(\mathrm{K}+)$ concentration. Data are expressed as mean \pm SEM. Statistical significance was declared when ${ }^{*} p<0.05,{ }^{* *} p<0.01,{ }^{* * *} p<0.001$ between basal and each time point; $n=$ seven animals, except for recovery period, $n=$ four animals.

\subsection{Electrochemical Sensors}

\subsection{1. $\mathrm{pO}_{2}$ Electrochemical Sensors}

A total of nine $\mathrm{pO}_{2}$ electrochemical sensors were included for the final analysis. A description of the signal detected by the $\mathrm{pO}_{2}$ electrochemical sensor during the different stages of the study is detailed in Figure 3a. In the 50\% occlusion stage, the electric signal presented an increase that was more marked in the $100 \%$ occlusion stage with an overall signal increase at the end of the $100 \%$ occlusion phase of $42.31 \%$ that was similar to the $\mathrm{pO}_{2}$ decrease observed with standard gasometry (56.57\% decrease). In the recovery stage, during the first $10 \mathrm{~min}$, the sensor signal decreased following the $\mathrm{pO}_{2}$ change observed by the standard EPOC equipment in this stage, reaching similar values of the basal period. 

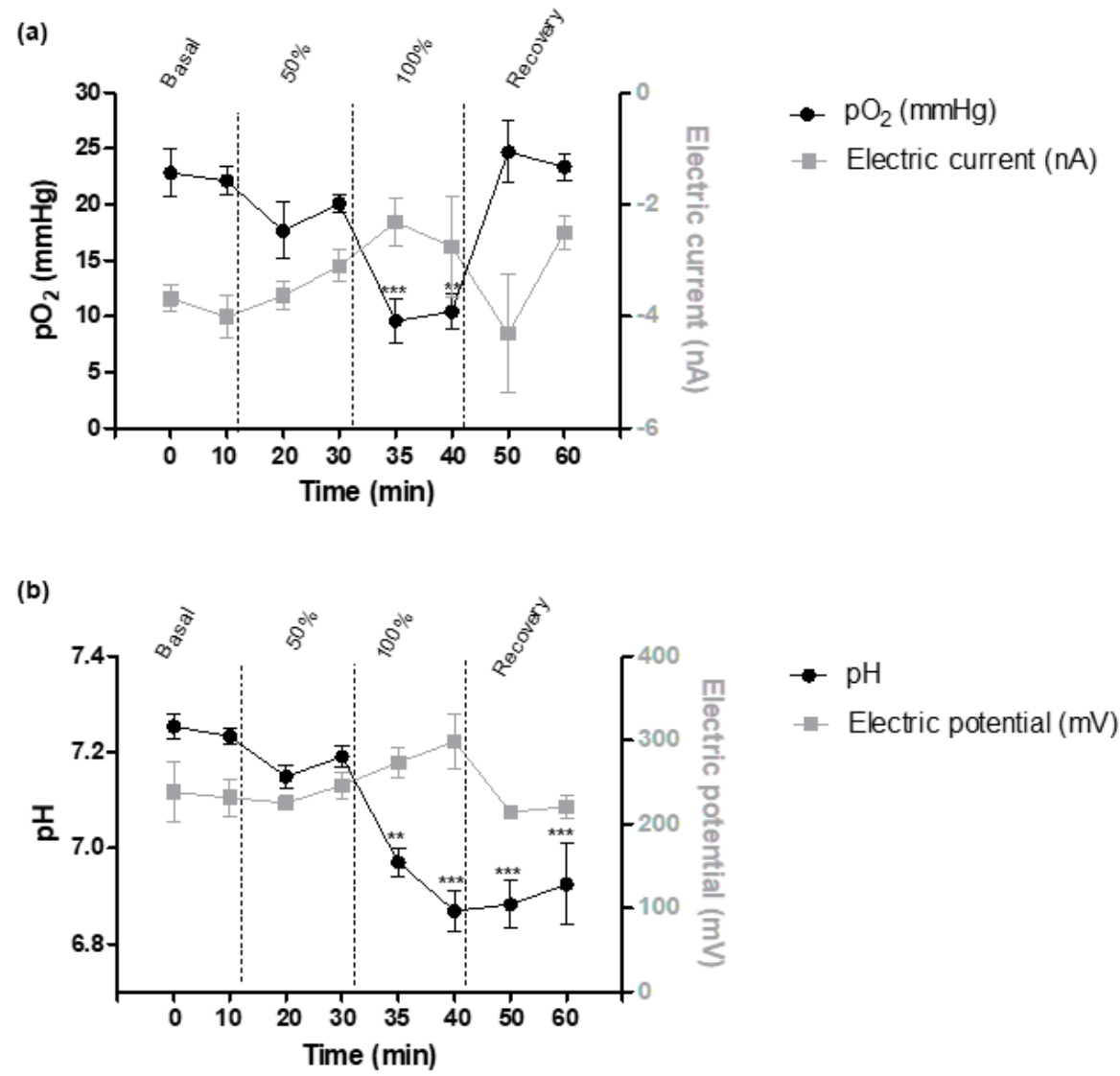

Figure 3. (a) Evolution of partial pressure of oxygen $\left(\mathrm{pO}_{2}\right)$ (measured by arterial gasometry) and electric current (nA) (measured by oxygen electrochemical sensors); (b) evolution of $\mathrm{pH}$ (measured by arterial gasometry) and electric potential $(\mathrm{mV})$ (measured by $\mathrm{pH}$ electrochemical sensors) during the occlusion protocol. Data are expressed as mean \pm SEM. For $\mathrm{pO}_{2}$ sensing, nine electrochemical sensors; for $\mathrm{pH}$ sensing, 10 electrochemical sensors. Statistical significance was declared when ** $p<0.01$ and ${ }^{* * *} p<0.001$ between basal and each time point.

\subsection{2. $\mathrm{pH}$ Electrochemical Sensors}

A total of $10 \mathrm{pH}$ electrochemical sensors were included for $\mathrm{pH}$ recordings in tissue. A description of the signal detected by the $\mathrm{pH}$ electrochemical sensor during the different stages of the study is detailed in Figure 3b. In the 50\% occlusion stage, the electric signal presented an increase that was more marked in the $100 \%$ occlusion stage with an overall signal increase of $28.67 \%$ that was higher than $\mathrm{pH}$ change shown by EPOC $(5.03 \%$ of decrease). In the recovery stage, electric signal reached similar values to those found in the basal period.

All raw data obtained from the acid-based metabolites and readings of the electrochemical sensors are detailed in Supplementary Tables S1-S5.

\subsection{Fetal Hemodynamics}

Table 1 depicts Doppler results of the different phases of the study available for six animals. In the $50 \%$ occlusion stage, only UV diameter presented a significant decrease in comparison with basal stage. The most relevant changes were observed in the $100 \%$ occlusion stage, where UV blood flow velocity and UV diameter presented a significant decrease. 
Table 1. Evolution of Doppler hemodynamics during the UCO protocol.

\begin{tabular}{ccccc}
\hline Stage & Basal & $\begin{array}{c}\mathbf{5 0 \%} \\
\text { Occlusion }\end{array}$ & $\begin{array}{c}\mathbf{1 0 0 \%} \\
\text { Occlusion }\end{array}$ & Recovery \\
\hline FHR $(\mathrm{bpm})$ & $135.8(5.50)$ & $129.3(13.68)$ & $116.8(21.79)$ & $115.7(12.83)$ \\
\hline UAPI & $1.35(0.23)$ & $1.48(0.23)$ & $3.09(1.24)$ & $0.99(0.06)$ \\
\hline DVPI & $0.52(0.09)$ & $0.46(0.06)$ & $1.79(0.47)$ & $2.92(2.37)$ \\
\hline UV flow velocity $(\mathrm{cm} / \mathrm{s})$ & $16.38(3.61)$ & $18.09(6.79)$ & $-17.70(10.96)^{*}$ & $19.78(7.96)$ \\
\hline UV diameter $(\mathrm{mm})$ & $5.77(0.41)$ & $3.79(0.22)^{* *}$ & $2.95(0.51)^{* * *}$ & $5.77(0.31)$ \\
\hline
\end{tabular}

Statistical significance was declared when ${ }^{*} p<0.05$, $^{* *} p<0.01$ and ${ }^{* * *} p<0.001$ between basal and each stage Values are mean and standard error of mean. FHR - fetal heart rate; UAPI—pulsatility index of the umbilical artery; DVPI-pulsatility index of the ductus venosus; UV—umbilical vein.

\subsection{Correlation Results}

Correlation results are shown in Table 2. A high correlation was observed between the oxygen metabolite with the current signal detected by the oxygen sensor, although without reaching statistical significance. The correlation between $\mathrm{pH}$ and the electrochemical signal was weaker. On the contrary, a significant correlation between sensor signaling and Doppler evaluation was observed in the $\mathrm{pH}$ sensing. The correlation was significant especially with the UAPI and the UV blood flow.

Table 2. Correlation results.

(a) Correlation between electrochemical sensing results and $\mathrm{pO}_{2}$ and $\mathrm{pH}$ results

\begin{tabular}{ccc}
\hline $\begin{array}{c}\text { Electrochemical Signal } \\
\text { (nA or mV) }\end{array}$ & $\mathrm{pO}_{2}$ & $\mathrm{pH}$ \\
\hline $\mathrm{nA}$ & 0.82 & - \\
\hline $\mathrm{mV}$ & - & 0.37 \\
\hline
\end{tabular}

(b) Correlation between electrochemical sensing results and Doppler results

\begin{tabular}{ccccc}
\hline $\begin{array}{c}\text { Electrochemical Signal } \\
\text { (nA or } \mathbf{~ m V )}\end{array}$ & UAPI & DVPI & $\begin{array}{c}\text { UV } \\
\text { Diameter }\end{array}$ & $\begin{array}{c}\text { UV Blood Flow } \\
\text { Velocity }\end{array}$ \\
\hline $\mathrm{nA}$ & 0.77 & 0.13 & 0.71 & 0.79 \\
\hline $\mathrm{mV}$ & $0.99^{*}$ & 0.01 & 0.68 & $0.95^{*}$ \\
\hline
\end{tabular}

Data are expressed as $\mathrm{r}$ squared. Statistical significance was declared when * $p<0.05$. "possible; UAPI—pulsatility index of the umbilical artery; DVPI—pulsatility index of the ductus venosus; UVumbilical vein.

\subsection{Histology}

Muscular tissue surrounding the implantable sensor insertion area showed no signs of alteration around the site of implantation. Normal muscular parenchyma, with neither inflammatory reaction nor deposition of collagen fibers, was observed (Figure 4). 
(a)

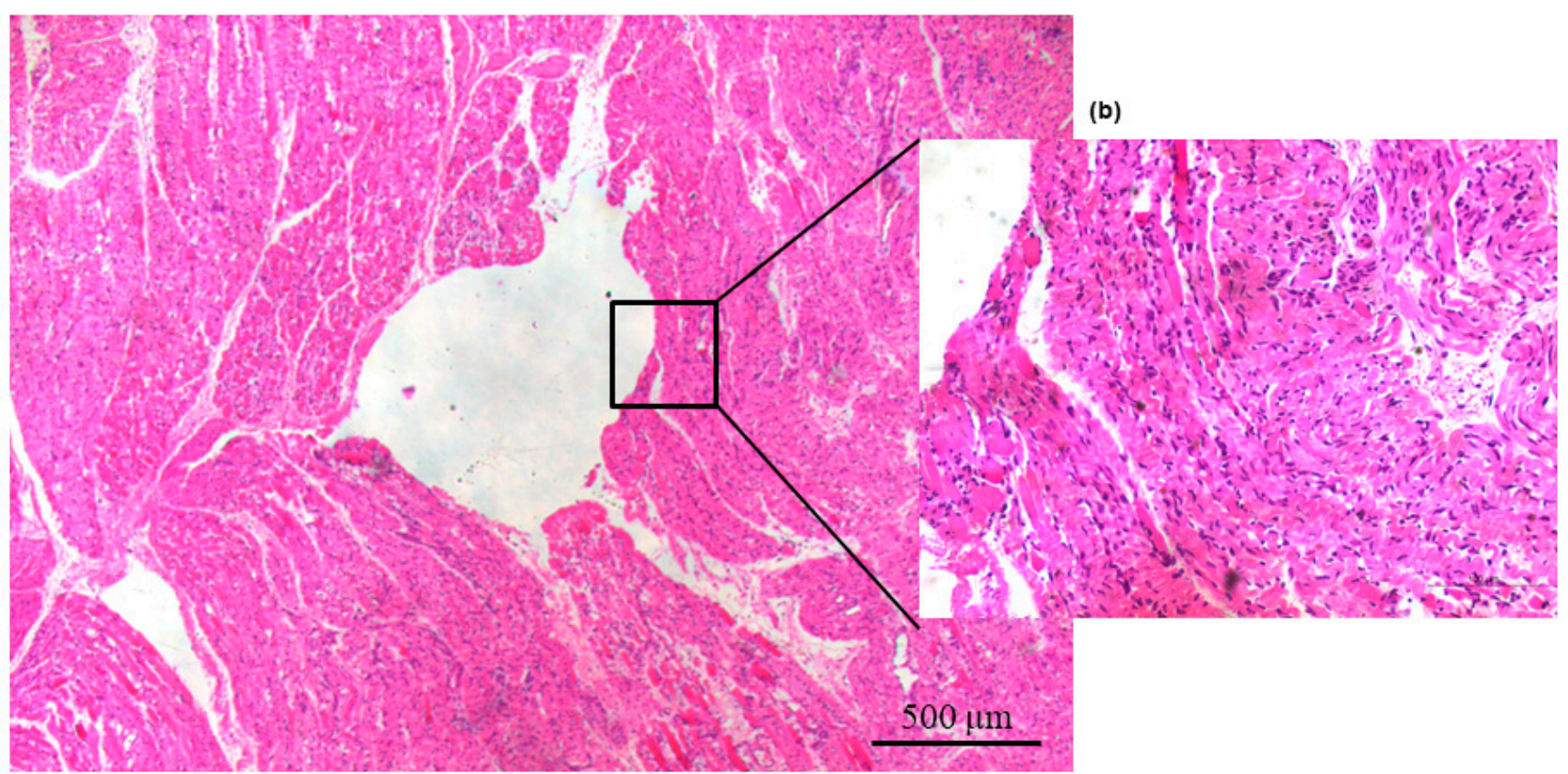

Figure 4. Images of (a) hematoxylin/eosin and (b) Masson trichrome staining of sites of implantation. (a) Normal muscular parenchyma delimitating the area of implantation without any inflammatory reaction at $5 \times$; (b) Normal muscular parenchyma delimitating the area of implantation without any deposit of collagen fibers at $20 \times$.

\section{Discussion}

In this study, we report for the first time a capability to monitor prenatal asphyxia continuously with specific miniaturized multiparametric electrochemical sensors for $\mathrm{pH}$ and $\mathrm{pO}_{2}$ in fetal sheep.

\subsection{Acid-Based Metabolites}

Our results of partial occlusion (50\%) are in accordance with other models with partial UCO in fetal sheep at 130 days of gestation [16], with a decrease of 4 units in the arterial $\mathrm{pO}_{2}$ and $0.04 \mathrm{in} \mathrm{pH}$. These modest falls in the acid-based metabolites contrasted with the marked alterations obtained in the $100 \%$ occlusion stage. In comparison with the basal level, significant decreases of arterial $\mathrm{pO}_{2}(22.64 \mathrm{mmHg}$ to $11.73 \mathrm{mmHg})$ and fetal $\mathrm{pH}$ (7.24 to 6.86) were obtained. These pronounced alterations are comparable with other severe UCO models in fetal sheep between 124 and 131 days of gestation [17-19].

When comparing the pattern of changes for each metabolite, the most pronounced and fastest change was the decrease in the $\mathrm{pO}_{2}$, whereas $\mathrm{pH}$ alterations were less pronounced and more gradual. During the recovery, basal values were only reached in $\mathrm{pO}_{2}$ whereas changes in the remaining parameters did not fully revert to the basal values. This can be explained by the fact that changes in $\mathrm{pH}$, lactate, $\mathrm{HCO}_{3}{ }^{-}$, and $\mathrm{K}+$ are produced by the cellular metabolism in response to the decrease in oxygen levels in the blood. This response includes several changes at the cellular level making it a slower and more gradual response in comparison with the oxygen levels [20]. In fact, in some of the cases, the $\mathrm{pH}$ persisted below fetal viability (ranging around 6.69 and 6.77) leading to fetal death. These differences in the pattern of changes for each parameter have already been documented in previous studies with similar methodology [17-19].

\subsection{Electrochemical Sensors}

Overall, the oxygen sensor detected $\mathrm{pO}_{2}$ changes induced by the occlusion protocol in the umbilical cord. The signal of the oxygen sensor increased in inverse proportion to the decrease in $\mathrm{pO}_{2}$, suggesting a good performance of the sensor in detecting pronounced changes in $\mathrm{pO}_{2}$. Additionally, in the recovery stage, the electrochemical signal returned to 
basal levels following the changes obtained in the blood test analyses. Previous studies with electrochemical sensors were designed similar to those used in this study demonstrated different sensibilities. In an adult rabbit model with a ventilatory hypoxia induction, the $\mathrm{pO}_{2}$ electrochemical sensor inserted in the skeletal muscle presented lower sensibility in comparison with the sensibility described here [12]. These differences may be explained with different reasons. Firstly, the animal model used may have an influence. In the previous study, acute ventilatory hypoxia was induced in an adult rabbit, in contrast with the UCO model induced in fetal sheep presented here. Secondly, basal oxygen levels differ between adults and fetuses since the amount of oxygen that reaches the fetal circulation is reduced, meaning that even small changes of oxygen level can cause a change from normoxia to hypoxia. Thirdly, differences in oxygen-hemoglobin and oxygen-myoglobin dissociation curves between fetuses and adults can explain the difference in function of the sensors. Fetal hemoglobin and myoglobin from skeletal muscle tissue have a higher affinity for oxygen than adult hemoglobin and myoglobin, becoming more saturated at the same oxygen levels [21,22]. Finally, differences in the design of the sensors with longer wires may also partially explain these results.

Regarding $\mathrm{pH}$ sensor, the $\mathrm{pH}$ sensor detected the pattern of $\mathrm{pH}$ blood changes induced by the umbilical occlusion protocol, although its sensibility was weaker in comparison with the oxygen sensor. During the occlusion period, an increase in the signal from the $\mathrm{pH}$ electrochemical sensor was obtained following the pattern of $\mathrm{pH}$ reduction in the blood test analysis, suggesting a good functionality of the sensor. Regarding the lower sensibility in comparison with the oxygen sensor, this could be due to the lower percentage of $\mathrm{pH}$ change during the UCO in comparison with the $\mathrm{pO}_{2}$ values. In addition, the decrease in $\mathrm{pH}$ due to the lack of oxygen requires the activation of the cellular anaerobic metabolism with the subsequent lactic acid formation. In this sense, tissue acidosis is more delayed and slower than the marked changes in the oxygen levels that only depend on the blockage of the oxygenated blood flowing through the umbilical cord. In the recovery stage, although the $\mathrm{pH}$ began to progressively increase, the signal measured with the electrochemical sensor implanted in the muscular tissue dropped more steeply during the first $10 \mathrm{~min}$, reaching similar values to the basal period. On the other hand, standard $\mathrm{pH}$ equipment used to analyze blood did not reach baseline values during the study. This could be because acidosis produced in the tissue by cells affected by hypoxia takes some time to reach the blood flow, producing a delay in the response in blood-based measurement. Finally, as observed with the oxygen sensor, the sensibilities observed for the $\mathrm{pH}$ sensing differ from a previous study [12]. Again, these differences may be explained by the same three reasons as discussed above on the differences observed with the oxygen sensor.

Finally, the most relevant correlations were detected between Doppler parameters and electrochemical sensing, especially between UAPI and UV blood flow with the $\mathrm{pH}$ electrochemical sensor signal. On the contrary, a weaker correlation was detected between Doppler parameters and $\mathrm{pO}_{2}$ levels. Along this line, previous papers detected a significant association between lower levels of $\mathrm{pH}$ and Doppler changes [23]. Both $\mathrm{pH}$ and Doppler changes reflect a more stationary stage in the hypoxia-acidosis process, whereas $\mathrm{pO}_{2}$ changes are more dynamic and reversible [24].

\subsection{Strengths and Limitations}

Two of the main strengths of this study are the size of the electrochemical sensors and the added value of their multiparametric design. Their minimally invasive size means they can potentially be inserted by a needle that can be later withdrawn, leaving the sensor in the fetal skeletal muscle. Additionally, the multiparametric electrochemical sensors offer dynamical information regarding the different stages of tissue ischemia. Thanks to the oxygen sensor the device could detect an initial lack of oxygen, whereas the $\mathrm{pH}$ sensor may detect the tissue's response to anaerobic conditions resulting in tissue acidosis. This information would be of value in clinical practice since it would give reliable information regarding the metabolic status of the fetus in real-time. Finally, the transferability of this 
research to clinics is high since the animal model used (pregnant sheep model) has been extensively used due to its similar fetal size and suitability to reproduce well the fetal hypoxic conditions found in human pregnancy [25].

This study has also some limitations. First, the electrochemical sensors studied here were able to detect changes at the $100 \%$ occlusion stage, while the acid-base changes were more pronounced. The detection of acid-based metabolites in fetal tissue is a challenge itself as the amount of oxygen that reaches the fetal circulation is reduced in comparison with adults, meaning that the difference in oxygen concentration between basal and hypoxia is very small [26]. In this regard, future directions of this work would be to improve the sensibility of these miniaturized electrochemical sensors in order to be able to detect more subtle hypoxia-acidosis changes such as those that occur in the $50 \%$ occlusion stage, more comparable to mild and chronic hypoxia-ischemia episodes. Finally, we did not assess long-term functionality testing; further studies including follow-up evaluation would be required. Finally, future sensor design focused on the telemetric system would give more functions to the whole system allowing real-time monitoring.

\section{Conclusions}

This study provides the first evidence showing the application of miniaturized multiparametric electrochemical sensors, capable of detecting changes in oxygen and $\mathrm{pH}$ in skeletal muscular tissue in fetal sheep. The use of such devices would open new applications in the field of fetal monitoring of pregnancies at high risk of fetal hypoxia-acidosis. Further research is required to advance in the adaptation and refinement of these electrochemical sensors to render them suitable for clinical testing.

Supplementary Materials: The following are available online at https:/ / www.mdpi.com/article/10 .3390 /biomedicines9101344/s1, Figure S1: Scheme of miniaturized electrochemical sensors: $\mathrm{pH}$ and $\mathrm{pO}_{2}$; Table S1: Acid-based metabolite results during the umbilical cord occlusion protocol; Table S2: Electric current measured by oxygen electrochemical sensors during the umbilical cord occlusion protocol; Table S3: Oxygen and $\mathrm{pH}$ results during the umbilical cord occlusion protocol from the animals used for oxygen electrochemical sensor evaluation; Table S4: Electric potential measured by $\mathrm{pH}$ electrochemical sensors during the umbilical cord occlusion protocol; Table S5: Oxygen and $\mathrm{pH}$ results during the umbilical cord occlusion protocol from the animals used for $\mathrm{pH}$ electrochemical sensor evaluation.

Author Contributions: Conceptualization, M.I., E.G., E.E., J.S. and M.M.; methodology, S.B. and L.P.; software, S.D. and L.R.; formal analysis, M.I. and L.P.; investigation, S.B., M.I., L.P., S.D. and L.R.; resources, N.P.-H.; writing-original draft preparation, M.I. and L.P.; writing-review and editing, E.E. and M.M.; supervision, E.G. and J.S. All authors have read and agreed to the published version of the manuscript.

Funding: This research was funded by CELLEX FOUNDATION. This work was financially supported by The Cellex Foundation and the Agència de Gestió d'Ajuts Universitaris i de Recerca (Grant 2017 SGR 1531). Additionally, E.E. has received support from the Departament de Salut (Grant SLT008/18/00156). The Nanobioengineering group at the Institute of Bioengineering of Catalonia (IBEC) has received support from the Commission for Universities and Research of the Department of Innovation, Universities, and Enterprise of the Generalitat de Catalunya (No. 2017 SGR 1079) and is part of the CERCA Programme / Generalitat de Catalunya and is supported by the Severo Ochoa programme of the Spanish Ministry of Science and Competitiveness (Grant SEV-2014-0425 (2015-2019)). CIBER-BBN is an initiative funded by the VI National R\&D\&i Plan 2008-2011, Iniciativa Ingenio 2010, Consolider Program, CIBER Actions and financed by the Instituto de Salud Carlos III with assistance from the European Regional Development Fund. L.R. would also like to acknowledge her support within the BEST Postdoctoral Programme, funded by the European Commission under Horizon 2020's Marie Skłodowska-Curie Actions COFUND scheme (Grant Agreement No. 712754).

Institutional Review Board Statement: Animal handling and all experimental procedures were performed in accordance with applicable regulation and guidelines and with the approval of the Animal Experimental Ethics Committee of the Universitat de Barcelona on 12 Desember 2017 (Ref. 214.17 and the competent authority Generalitat de Catalunya (Ref. 9645)). 
Informed Consent Statement: Not applicable.

Data Availability Statement: All data generated or analyzed during this study are included in this published article [and its Supplementary Materials].

Acknowledgments: The authors acknowledge Project PI18/01763, funded by Instituto de Salud Carlos III and co-funded by the European Union (ERDF 'A way to make Europe') and also the animal facility of the Institut d'Investigació Biomédica de Bellvitge (IDIBELL) for their help in maintaining animal welfare.

Conflicts of Interest: The authors declare no conflict of interest.

\section{References}

1. McGuire, W. Perinatal asphyxia. BMJ Clin. Evid. 2007, 2007, 0320.

2. Rainaldi, M.A.; Perlman, J.M. Pathophysiology of Birth Asphyxia. Clin. Perinatol. 2016, 43, 409-422. [CrossRef] [PubMed]

3. Herrera, C.A.; Silver, R.M. Perinatal Asphyxia from the Obstetric Standpoint: Diagnosis and Interventions. Clin. Perinatol. 2016, 43, 423-438. [CrossRef]

4. Baschat, A.A. Arterial and venous Doppler in the diagnosis and management of early onset fetal growth restriction. Early Hum. Dev. 2005, 81, 877-887. [CrossRef] [PubMed]

5. Figueras, F.; Gratacós, E. Update on the Diagnosis and Classification of Fetal Growth Restriction and Proposal of a Stage-Based Management Protocol. Fetal Diagn. Ther. 2014, 36, 86-98. [CrossRef] [PubMed]

6. Garite, T.J.; Dildy, G.A.; McNamara, H.; Nageotte, M.P.; Boehm, F.H.; Dellinger, E.H.; Knuppel, R.A.; Porreco, R.P.; Miller, H.S.; Sunderji, S.; et al. A multicenter controlled trial of fetal pulse oximetry in the intrapartum management of nonreassuring fetal heart rate patterns. Am. J. Obstet. Gynecol. 2000, 183, 1049-1058. [CrossRef]

7. Fong, D.D.; Knoesen, A.; Motamedi, M.; O'Neill, T.; Ghiasi, S. Recovering the fetal signal in transabdominal fetal pulse oximetry. Smart Health 2018, 9-10, 23-36. [CrossRef]

8. Devlieger, R.; Gratacós, E.; Wu, J.; Yesildaglar, N.; Ghysel, C.; Barki, G.; Deprest, J. Continuous Monitoring of Fetal pH, pO 2 and $\mathrm{pCO}_{2}$ Using a Fiberoptic Multiparameter Sensor in Animal Models Reproducing in utero Conditions. Fetal Diagn. Ther. 2000, 15, 127-131. [CrossRef]

9. Nickelsen, C.; Thomsen, S.G.; Weber, T. Continuous acid-base assessment of the human fetus during labour by tissue pH and transcutaneous carbon dioxide monitoring. BJOG Int. J. Obstet. Gynaecol. 1985, 92, 220-225. [CrossRef]

10. Nickelsen, C.; Weber, T. The current status of intrapartum continuous fetal tissue $\mathrm{pH}$ measurements. J. Périnat. Med. 1991, 19, 87-92. [CrossRef]

11. Chatterjee, M.S.; Hochberg, H.M. Continuous intrapartum measurement of tissue $\mathrm{pH}$ of the human fetus using newly developed techniques. J. Périnat. Med. 1991, 19, 93-96. [CrossRef] [PubMed]

12. Pla, L.; Berdún, S.; Mir, M.; Rivas, L.; Miserere, S.; Dulay, S.; Samitier, J.; Eixarch, E.; Illa, M.; Gratacós, E. Non-invasive monitoring of $\mathrm{pH}$ and oxygen using miniaturized electrochemical sensors in an animal model of acute hypoxia. J. Transl. Med. 2021, 19, 1-11. [CrossRef] [PubMed]

13. Rivas, L.; Dulay, S.; Miserere, S.; Pla, L.; Marin, S.B.; Parra, J.; Eixarch, E.; Gratacós, E.; Illa, M.; Mir, M.; et al. Micro-needle implantable electrochemical oxygen sensor: Ex-vivo and in-vivo studies. Biosens. Bioelectron. 2020, 153, 112028. [CrossRef] [PubMed]

14. Dulay, S.; Rivas, L.; Miserere, S.; Pla, L.; Berdún, S.; Parra, J.; Eixarch, E.; Gratacós, E.; Illa, M.; Mir, M.; et al. in vivo Monitoring with micro-implantable hypoxia sensor based on tissue acidosis. Talanta 2021, 226, 122045. [CrossRef] [PubMed]

15. Gonzalez-Bulnes, A.; Astiz, S.; H. Parraguez, V.; Garcia-Contreras, C.; Vazquez-Gomez, M. Empowering Translational Research in Fetal Growth Restriction: Sheep and Swine Animal Models. Curr. Pharm. Biotechnol. 2016, 17, 848-855. [CrossRef] [PubMed]

16. Giussani, D.A.; Unno, N.; Jenkins, S.L.; Wentworth, R.A.; Derks, J.B.; Collins, J.H.; Nathanielsz, P. Dynamics of cardiovascular responses to repeated partial umbilical cord compression in late-gestation sheep fetus. Am. J. Physiol. Circ. Physiol. 1997, 273, H2351-H2360. [CrossRef]

17. Fujii, E.Y.; Takahashi, N.; Kodama, Y.; Roman, C.; Ferriero, N.M.; Parer, J.T. Hemodynamic changes during complete umbilical cord occlusion in fetal sheep related to hippocampal neuronal damage. Am. J. Obstet. Gynecol. 2003, 188, 413-418. [CrossRef]

18. Lotgering, F.K.; Bishai, J.M.; Struijk, P.C.; Blood, A.; Hunter, C.; Power, G.G.; Longo, L.D. Ten-minute umbilical cord occlusion markedly reduces cerebral blood flow and heat production in fetal sheep. Am. J. Obstet. Gynecol. 2003, 189, 233-238. [CrossRef]

19. Yawno, T.; Castillo-Melendez, M.; Jenkin, G.; Wallace, E.M.; Walker, D.W.; Miller, S.L. Mechanisms of Melatonin-Induced Protection in the Brain of Late Gestation Fetal Sheep in Response to Hypoxia. Dev. Neurosci. 2012, 34, 543-551. [CrossRef]

20. Michiels, C. Physiological and Pathological Responses to Hypoxia. Am. J. Pathol. 2004, 164, 1875-1882. [CrossRef]

21. Bank, A. Regulation of human fetal hemoglobin: New players, new complexities. Blood 2006, 107, 435-443. [CrossRef] [PubMed]

22. Longo, L.; Koos, B.; Power, G. Fetal myoglobin: Quantitative determination and importance for oxygenation. Am. J. Physiol. Content 1973, 224, 1032-1036. [CrossRef]

23. Bilardo, C.M.; Nicolaides, K.; Campbell, S. Doppler measurements of fetal and uteroplacental circulations: Relationship with umbilical venous blood gases measured at cordocentesis. Am. J. Obstet. Gynecol. 1990, 162, 115-120. [CrossRef] 
24. Omo-Aghoja, L. Maternal and fetal acid-base chemistry: A major determinant of perinatal outcome. Ann. Med. Health Sci. Res. 2014, 4, 8-17. [CrossRef]

25. Morrison, J.L.; Berry, M.J.; Botting, K.J.; Darby, J.R.T.; Frasch, M.G.; Gatford, K.L.; Giussani, D.; Gray, C.L.; Harding, R.; Herrera, E.A.; et al. Improving pregnancy outcomes in humans through studies in sheep. Am. J. Physiol. Integr. Comp. Physiol. 2018, 315, R1123-R1153. [CrossRef] [PubMed]

26. Morton, S.U.; Brodsky, D. Fetal Physiology and the Transition to Extrauterine Life. Clin. Perinatol. 2016, 43, 395-407. [CrossRef] [PubMed] 